\title{
QUADRATIC VARIATION OF POTENTIALS AND HARMONIC FUNCTIONS
}

\author{
BY \\ GUNNAR A. BROSAMLER
}

\begin{abstract}
We prove the existence of a finite quadratic variation for stochastic processes $u(Y)$, where $Y$ is Brownian motion on a Green domain of $R^{n}$, stopped upon reaching the Martin boundary, and $u$ is a positive superharmonic function on the domain. As by-products we have results which are also of interest from a nonprobabilistic point of view.
\end{abstract}

1. Introduction and summary. Let $X=\left\{X_{t}, 0 \leqq t \leqq T<\infty\right\}$ be a stochastic process with a.e. continuous sample paths; let $\pi_{n}$ be a sequence of partitions of $[0, T]$ given by $0=t_{0}^{(n)}<t_{1}^{(n)}<\cdots<t_{k_{n}}^{(n)}=T$. Let $S^{2}\left(X, \pi_{n}\right)=\sum_{j=0}^{k_{n}-1}\left(X_{t_{+1}^{(n)}}-X_{t_{j}^{(n)}}\right)^{2}$. Then it may be important to know the asymptotic behaviour of the random variable $S^{2}\left(X, \pi_{n}\right)$, if $\left\|\pi_{n}\right\|=\max _{j}\left(t_{j+1}^{(n)}-t_{j}^{(n)}\right) \rightarrow 0$. If a limit exists in some sense it is called the quadratic variation of $X$.

This problem was studied first by $\mathrm{P}$. Lévy in the case where $X$ is Brownian motion on $[0, T]$. Lévy obtained $L_{1}$-convergence of $S^{2}\left(X, \pi_{n}\right)$ if $\left\|\pi_{n}\right\| \rightarrow 0$ and a.e. convergence if either $\left\{\pi_{n}\right\}$ is monotone and $\left\|\pi_{n}\right\| \rightarrow 0$ or if $\sum\left\|\pi_{n}\right\|<\infty$. In this case the limit is the constant $T$. Since under very general assumptions continuous martingales can be obtained as images of Brownian motion under a random time change [2] it is suggestive to expect similar results for continuous martingales. In [7], the problem of quadratic variation was studied for $L_{2}$-martingales which can be written as stochastic integrals with respect to Brownian motion. In [4] $L_{1}$ convergence of $S^{2}\left(X, \pi_{n}\right)$ was proved for the wider class of continuous $L_{2}$-martingales $X$, if $\left\|\pi_{n}\right\| \rightarrow 0$. In that paper it was also pointed out that the increasing process $A$ of the Doob decomposition of the submartingale $X^{2}$ plays an important role. The most recent work is a paper by Millar [6] which discusses convergence of $S^{2}\left(X, \pi_{n}\right)$ in probability of $L_{1}$-bounded martingales. This includes of course martingales for which $X^{2}$ may fail to be a submartingale because $E X_{t}^{2}=\infty$. Under stronger assumptions Millar obtains convergence of $S\left(X, \pi_{n}\right)$ in $L_{p}$-norm.

It is the purpose of this paper to discuss the quadratic variation of a process $\left\{u\left(Y_{t}\right), t \geqq 0\right\}$ where

(1) $Y$ is Brownian motion on some Green domain $\Omega \subseteq R^{n}$, stopped upon reaching $\partial \Omega$, the Martin boundary of $\Omega$,

Received by the editors January 9, 1969.

AMS Subject Classifications. Primary 6062, 3115.

Key Words and Phrases. Quadratic variation of martingales, classical probabilistic potential theory, additive functionals of Brownian motion, Ito formula.

Copyright (C) 1970, American Mathematical Society 
(2) $u$ is either a potential on $\Omega$ or the difference of two positive harmonic functions on $\Omega$, and is extended to $\partial \Omega$ by its fine boundary function $u^{*}$.

We shall show that a quadratic variation of the process $u(Y)$ always exists in the sense that $S^{2}\left(u(Y), \pi_{n}\right)$ converges in probability for very general $\left\{\pi_{n}\right\}$, and we shall compute the value of the limit. Under somewhat stronger assumptions we shall obtain convergence of $S\left(u(Y), \pi_{n}\right)$ a.e. and also in $L_{2}$-norm if $u$ is a potential and in $\boldsymbol{L}_{p}$-norm $(p>1)$ if $u$ is harmonic. In $\S 5$ we derive a generalized version of a formula of K. Ito.

For our proofs we shall use results in [4], [6] for martingales and make use of the theory of additive functionals for $n$-dimensional Brownian motion. This theory is presented for general Markov processes e.g. in [1].

The statements on a.e. convergence of $S\left(u(Y), \pi_{n}\right)$ will be obtained from a lemma on martingales whose derivation we give in $\$ 2$ and which strengthens a theorem in [7].

As by-products of our discussion we will have a few results which might also be interesting from a strictly nonprobabilistic point of view. Finally we remark that parts of our theorems have formulations in terms of general superharmonic functions.

ACKnowledgement. The author would like to thank Professor J. L. Doob for suggesting the problem and his kind interest during the preparation of this paper.

2. Three lemmas for martingales. Let $M=\left\{M_{t}, \mathfrak{F}_{t}, 0 \leqq t \leqq \infty\right\}$ be a martingale for which almost all sample paths are continuous and $E M_{\infty}^{2}<\infty$ and $\mathfrak{F}_{t}=\mathfrak{F}_{t^{+}}$. All processes will be assumed adapted to $\mathfrak{F}_{t}$. The submartingale $M^{2}$ is of class $(D)$ and moreover, because of the continuity of the $M$-paths, regular in the sense of [5]. Therefore $M^{2}$ has a Doob decomposition:

$$
M_{t}^{2}=M_{0}^{2}+N_{t}+A_{t}, \quad 0 \leqq t \leqq \infty,
$$

where $N_{t}$ is a continuous martingale and $A_{t}$ is a continuous nondecreasing process and $N_{0}=A_{0}=0$. The decomposition is unique.

Let $\left\{\pi_{n}\right\}$ be a sequence of partitions given by

$$
0=t_{0}^{(n)}<t_{1}^{(n)}<\cdots<t_{k_{n}}^{(n)}<\infty
$$

such that (1) $t_{k_{n}}^{(n)} \rightarrow \infty$, (2) $\left\|\pi_{n}\right\|=\max _{j}\left(t_{j+1}^{(n)}-t_{j}^{(n)}\right) \rightarrow 0$.

Let $S^{2}\left(M, \pi_{n}\right)=\sum_{j}\left(M_{t_{j+1}^{(n)}}-M_{t_{j}^{(n)}}\right)^{2}$.

The following lemma is essentially contained in [4].

LEMMA 2.1. If $M, A,\left\{\pi_{n}\right\}$ are as above, then $S^{2}\left(M, \pi_{n}\right) \rightarrow A_{\infty}$ in $L_{1}$-norm and hence in probability.

LEMMA 2.2. If $M, A, \pi_{n}$ are as above then as $0 \leqq s \leqq t \leqq \infty$,

(a) $E\left(M_{t}-M_{s}\right)^{2}=E\left(A_{t}-A_{s}\right)$, (b) $E\left(M_{t}-M_{s}\right)^{4} \leqq 30 E\left(A_{t}-A_{s}\right)^{2}$.

Proof. Assume first that $s=0, M_{0}=0$ a.e. In this case (a) follows from the definition of $A$ and (b) is essentially contained in Theorem 6.2 in [6]. We shall give 
here a different derivation of (b): By [2] we know that we can redefine $M$ in such a way that there is a one-dimensional Brownian motion $Y$ for which $A$ is a random time change and for which $Y_{A_{t}}=M_{t}$.

However:

$$
\left\{\frac{1}{6} Y_{t}^{4}-t Y_{t}^{2}+\frac{1}{2} t^{2}, \mathfrak{F}_{t}, t \geqq 0\right\}
$$

is a martingale as is easily seen from the fact that the function

$$
u(y, t)=\frac{1}{6} y^{4}-t y^{2}+\frac{1}{2} t^{2}
$$

is a solution of $\frac{1}{2} u_{y y}+u_{t}=0$. Therefore, for any stopping time $\tau$,

$$
\left\{\frac{1}{6} Y_{t \wedge \tau}^{4}-(t \wedge \tau) Y_{t \wedge \tau}^{2}+\frac{1}{2}(t \wedge \tau)^{2}, \mathfrak{F}_{t}, t \geqq 0\right\}
$$

is also a martingale. Hence

$$
\frac{1}{6} E Y_{t \wedge \tau}^{4}+\frac{1}{2} E(t \wedge \tau)^{2}=E(t \wedge \tau) Y_{t \wedge \tau}^{2}
$$

where the expectations are finite. Applying the Schwarz inequality, we obtain:

$$
\left(E Y_{t \wedge \tau}^{4}\right)^{2}-30 E Y_{t \wedge \tau}^{4} \cdot E(t \wedge \tau)^{2}+9\left\{E(t \wedge \tau)^{2}\right\}^{2} \leqq 0,
$$

which implies $E Y_{t \wedge \tau}^{4} \leqq 30 E(t \wedge \tau)^{2}$. Letting $t \uparrow \infty$ we obtain by Fatou's Lemma and the monotone convergence theorem

$$
E Y_{\tau}^{4} \leqq 30 E \tau^{2} .
$$

Letting $\tau=A_{t}$, (b) follows.

The general case can be reduced to the one just considered: If we define $M_{t}^{\prime}=M_{t+s}-M_{s}, \mathfrak{F}_{t}^{\prime}=\mathfrak{F}_{t+s}$, then $\left\{M_{t}^{\prime}, \mathfrak{F}_{t}^{\prime}, 0 \leqq t \leqq \infty\right\}$ is a martingale for which $M_{0}^{\prime}=0$ a.e. The fact that $\left\{M_{t}^{2}-A_{t}, \mathfrak{F}_{t}, 0 \leqq t \leqq \infty\right\}$ is a martingale implies that $\left\{M_{t}^{\prime 2}-A_{t}^{\prime}, \mathfrak{F}_{t}^{\prime}, 0 \leqq t \leqq \infty\right\}$ is a martingale for $A_{t}^{\prime}=A_{t+s}-A_{s}$. Hence the increasing process in the Doob decomposition of $M_{t}^{\prime 2}$ is $A_{t}^{\prime}$.

Lemma 2.3. Let $M$ and $A$ be as above. Let a.e. $A \cdot(\omega)$ be absolutely continuous with respect to Lebesgue measure and let $\int_{0}^{\infty} E\left(\dot{A}_{t}^{2}\right) d t<\infty$. If $\left\{\pi_{n}\right\}$ is a sequence of partitions such that $t_{k_{n}}^{(n)} \rightarrow \infty$ and $\sum\left\|\pi_{n}\right\|<\infty$, then $S^{2}\left(M, \pi_{n}\right) \rightarrow A_{\infty}$ a.e.

Proof. (a) It is easy to see that $E S^{2}\left(M, \pi_{n}\right)=E A_{t_{k n}}^{(n)}$.

(b) We show now that

$$
E\left\{S^{2}\left(M, \pi_{n}\right)-A_{t_{k n}^{(n)}}\right\}^{2} \leqq 31 \sum_{j} E\left(A_{t_{j+1}^{(n)}}-A_{t_{j}^{(n)}}\right)^{2} .
$$

The left side equals

$$
\begin{aligned}
E\left[\sum _ { j = 0 } ^ { k _ { n } - 1 } \left\{\left(M_{t_{j+1}^{(n)}}-M_{t_{j}^{(n)}}\right)^{2}-\left(A_{t_{j+1}^{(n)}}-\right.\right.\right. & \left.\left.\left.A_{t_{j}^{(n)}}\right)\right\}\right]^{2} \\
& =\sum_{j=0}^{k_{n}-1} E\left\{\left(M_{t_{j+1}^{(n)}}-M_{t_{j}^{(n)}}\right)^{2}-\left(A_{t_{j+1}^{(n)}}-A_{t_{j}^{(n)}}\right)\right\}^{2} .
\end{aligned}
$$


The latter equation holds because the summands are orthogonal. This is derived as follows. If $t_{1}<t_{2}<t_{3}<t_{4}$, then

$$
\begin{aligned}
E\left\{\left(M_{t_{4}}-M_{t_{3}}\right)^{2}-\left(A_{t_{4}}-A_{t_{3}}\right)\right\}\left\{\left(M_{t_{2}}-M_{t_{1}}\right)^{2}-\left(A_{t_{2}}-A_{t_{1}}\right)\right\} \\
=E\left[\left\{\left(M_{t_{2}}-M_{t_{1}}\right)^{2}-\left(A_{t_{2}}-A_{t_{1}}\right)\right\} E\left\{\left(M_{t_{4}}-M_{t_{3}}\right)^{2}-\left(A_{t_{4}}-A_{t_{3}}\right) \mid \mathfrak{F}_{t_{3}}\right\}\right] .
\end{aligned}
$$

But the conditional expectation is 0 because $M_{t}^{2}-A_{t}$ is a martingale. Now

$$
E\left\{S^{2}\left(M, \pi_{n}\right)-A_{t_{k n}^{(n)}}\right\}^{2} \leqq \sum_{j=0}^{k_{n}-1}\left\{E\left(M_{t_{j+1}^{(n)}}-M_{t_{j}^{(n)}}\right)^{4}+E\left(A_{t_{j+1}^{(n)}}-A_{t_{j}^{(n)}}\right)^{2}\right\}
$$

and by Lemma 2.2

(c)

$$
\begin{aligned}
& \leqq 31 \sum_{j=0}^{k_{n}-1} E\left(A_{t_{j+1}^{(n)}}-A_{t_{j}^{(n)}}\right)^{2} \\
& P\left\{\left|S^{2}\left(M, \pi_{n}\right)-A_{t_{k}(n)}\right|>\varepsilon\right\} \leqq \frac{1}{\varepsilon^{2}} E\left\{S^{2}\left(M, \pi_{n}\right)-A_{t_{k_{n}}^{(n)}}\right\}^{2} \\
& \leqq \frac{31}{\varepsilon^{2}} \sum_{j=1}^{k_{n}-1} E\left(A_{t_{j+1}^{(n)}}-A_{t_{j}^{(n)}}\right)^{2}
\end{aligned}
$$

Now

$$
\left(A_{t_{j+1}^{(n)}}-A_{t_{j}^{(n)}}\right)^{2}=\left(\int_{t_{j}^{(n)}}^{t_{j+1}^{(n)}} A_{t} d t\right)^{2} \leqq\left(t_{j+1}^{(n)}-t_{j}^{(n)}\right) \int_{t_{j}^{(n)}}^{t_{j+1}^{(n)}} A_{t}^{2} d t
$$

and therefore

$$
P\left\{\left|S^{2}\left(M, \pi_{n}\right)-A_{t_{k_{n}}^{(n)}}\right|>\varepsilon\right\} \leqq \frac{31}{\varepsilon^{2}}\left\|\pi_{n}\right\| \int_{0}^{t_{k_{n}}^{(n)}} E\left(A_{t}^{2}\right) d t .
$$

Hence we have by the Borel-Cantelli lemma

$$
\left|S^{2}\left(M, \pi_{n}\right)-A_{t_{k_{n}}^{(n)}}\right| \rightarrow 0 \text { a.e. }
$$

and because $t_{k_{n}}^{(n)} \rightarrow \infty$ and therefore $A_{t_{k_{n}}^{(n)}} \rightarrow A_{\infty}$ a.e., we get $S^{2}\left(M, \pi_{n}\right) \rightarrow A_{\infty}$ a.e.

REMARK. The preceding lemma remains of course true if

(1) $M$ is a continuous $L_{2}$-martingale on the finite interval $[0, T]$ with a.e. absolutely continuous $A_{t}$ and $\int_{0}^{T} E\left(A_{t}^{2}\right) d t<\infty$.

(2) $\pi_{n}$ is a partition of $[0, T]$ such that $\sum\left\|\pi_{n}\right\|<\infty$. By Doob [3] the martingales of (1) are exactly the processes obtained as stochastic integrals $\int_{0}^{t} f_{s} d Y(s)$ where $Y$ is a 1-dimensional Brownian motion and $f$ is such that $\int_{0}^{T} E f_{t}^{4} d t<\infty$, both defined on a possibly enlarged probability space. The relation between $f$ and $A$ is given by $A_{t}=f_{t}^{2}$. For these stochastic integrals the assertion of Lemma 2.3 was proved in [7] under the stronger assumption that $\lim _{n \rightarrow \infty}\left\|\pi_{n}\right\| n^{2+\delta}=0$ for some $\delta>0$.

3. Quadratic variation of potentials. We introduce some notations to be used in this section and in $\S 4$ and $\S 5$. Let $\Omega$ be a Green domain $\subseteq R_{n}(n \geqq 2), \partial \Omega$ its Martin boundary and $g$ its Green function. Let $Y$ be Brownian motion on $\Omega \bigcup \partial \Omega$ stopped upon reaching $\partial \Omega ; P_{x}, E_{x}$ refer to this motion starting at $x$. As $\sigma$-fields $\mathfrak{F}_{t}$ (to be suppressed subsequently in the notation) we shall use $\mathscr{S}_{t^{+}}$, where the $\mathscr{S}_{t}$ 's 
are the $\sigma$-fields determined by the history of $Y$ up to time $t$. Let $\zeta$ be the first entry time of $Y$ into $\partial \Omega$. If $B$ is any open set, let $T_{B}$ denote the first entry time of $Y$ into $B$. If $f$ is an extended real-valued function on $\Omega$, we let $\Omega_{f}=\{x ; f(x)<\infty\}$. If $f$ is defined on $\Omega$ and has fine boundary function $f^{*}$, we extend $f$ to $\partial \Omega$ by $f^{*}$; and if $\left\{\pi_{n}\right\}$ is a sequence of partitions given by $0=t_{0}^{(n)}<t_{1}^{(n)}<\cdots<t_{k_{i}}^{(n)}<\infty$, we let

$$
S^{2}\left(f, \pi_{n}\right)=S^{2}\left(f(Y), \pi_{n}\right)=\sum_{j=0}^{k_{n}-1}\left\{f\left(Y_{t_{j+1}^{(n)}}\right)-f\left(Y_{t_{j}^{(n)}}\right)\right\}^{2} .
$$

Now let $p(x)=\int_{\Omega} g(x, y) \mu(d y)$ be a potential on $\Omega$. We recall the following wellknown properties (see e.g. [8], [9]):

(i) $p^{*} \equiv 0$.

(ii) $p=\sum_{k=1}^{\infty} p_{k}$, where the $p_{k}$ are continuous potentials which may assume the value $\infty$. (This follows e.g. from a theorem of Kishi which states that there is a sequence $F_{n} \uparrow \subseteq \Omega$ of closed sets such that $\mu\left(F_{n}^{c}\right) \downarrow 0$ and $\int_{F_{n}} g(\cdot, y) \mu(d y)$ are continuous potentials converging pointwise to $p$.)

(iii) $\Omega-\Omega_{p}$ is a polar set.

(iv) The first order derivatives of $p$ exist a.e. (w.r.t. Lebesgue measure) and are locally integrable. These derivatives are the derivatives of $p$ in distribution sense. Also in distribution sense: $\Delta p=-2 \mu$.

In view of (iii), $\Omega-\Omega_{p}$ is avoided by the $Y$-paths and we may and shall consider $Y$ as a process with state space $\Omega_{p}$. Let $\tau_{n}=T_{\{p>n\}}$. Then $\tau_{n}<\tau_{n+1}$ and if $x \in \Omega_{p}$, then $P_{x}$-a.e., $p\left(Y_{t}\right)$ is continuous (and finite) on $[0, \infty]$, which implies $P_{x}\left\{\tau_{n} \rightarrow \infty\right\}=1$.

The following two propositions answer the question of Doob decomposibility of the supermartingale $p(Y)$.

PROPOSITION 3.1. If $\mu$ lives on a polar set, then for all $n$ and all $x \in \Omega_{p}$, the processes $\left\{p\left(Y_{t \wedge \tau_{n}}\right), 0 \leqq t \leqq \infty, P_{x}\right\}$ are martingales.

Proof. We prove the theorem first for continuous $p$ (possibly assuming the value $\infty)$. Fix $x \in \Omega_{p}$. Since $\tau_{1}<\tau_{2}$ implies that $p\left(Y_{t \wedge \tau_{1}}\right)=p\left(Y_{t \wedge \tau_{2} \wedge \tau_{1}}\right)$, it is sufficient to prove the theorem for sufficiently large $n$. But for sufficiently large $n, x \in\{p<n\}$. The set $\{p<n\}$ is open and has $\mu$-measure 0 . This implies that $p$ is harmonic on $\{p<n\}$ and hence $\left\{p\left(Y_{t \wedge \tau_{n}}\right), 0 \leqq t \leqq \infty, P_{x}\right\}$ is a martingale.

For general $p$ let $p=\sum p_{k}$ where the $p_{k}$ are continuous and let $\tau_{n}^{(k)}=T_{\left\{p_{k}>n\right\}}$. Then for $x \in \Omega_{p}$,

$$
\begin{aligned}
E_{x} p\left(Y_{t \wedge \tau_{n}}\right) & =E_{x} \sum_{k} p_{k}\left(Y_{t \wedge \tau_{n}}\right)=\sum_{k} E_{x} p_{k}\left(Y_{t \wedge \tau_{n}}\right)=\sum_{k} E_{x} p_{k}\left(Y_{t \wedge \tau_{n}^{(k)} \wedge \tau_{n}}\right) \\
& =\sum_{k} p_{k}(x)=p(x) .
\end{aligned}
$$

Proposition 3.2. The following conditions are equivalent:

(1) $\mu$ does not charge any polar set.

(2) $\lim _{n \rightarrow \infty} n P_{x}\left\{\tau_{n}<\infty\right\}=0$ for all $x \in \Omega_{p}$.

(2') $\lim _{n \rightarrow \infty} n P_{x}\left\{\tau_{n}<\infty\right\}=0$ for some $x \in \Omega_{p}$. 
(3) There exists a continuous homogeneous additive functional $A$ of $Y$ such that $E_{x} A_{\infty}=p(x)$ for all $x \in \Omega_{p}$.

If one, and hence all, of these conditions are satisfied then:

(a) $A$ is determined uniquely (up to equivalence).

(b) If we let $M_{t}=p\left(Y_{t}\right)+A_{t}$, then the processes $\left\{M_{t}, 0 \leqq t \leqq \infty, P_{x}\right\}$ are martingales for all $x \in \Omega_{p}$.

(c) If $f \geqq 0$ is a measurable function on $\Omega$, then for $x \in \Omega_{p}$,

$$
E_{x} \int_{0}^{\zeta} f\left(Y_{s}\right) d A_{s}=\int_{\Omega} f(y) g(x, y) \mu(d y) .
$$

Proof. Let us say that $p$ fulfills condition (R) if the restriction of $p$ to $\Omega_{p}$ is a regular potential (of $(Y, 1)$ ) in the sense of [1], i.e. if $E_{x} p\left(Y_{T_{n}}\right) \rightarrow E_{x} p\left(Y_{T}\right)$ for all $x \in \Omega_{p}$ and stopping times $T_{n} \uparrow T$. By VI,T20 in [5] and the continuity of the $p(Y)$-paths, (2) and (R) are equivalent. By IV-T3.13, IV-T2.13, VI-T3.1 in [1], (R) implies (3), (a), (c). On the other hand (3) implies (R) by the remark preceding IV, D3.2 in [1] and (b) by a simple calculation. Since for Brownian motion polar sets and semipolar sets coincide, (1) implies (R) by VI,T3.5 in [1]. We want to show now that one of the equivalent conditions (R), (2), (3) implies (1). If the former conditions hold for $p$, we know from VI-T3.5 in [1] that $\mu$ does not charge any polar set $\subseteq \Omega_{p}$. In order to show that $\mu\{y ; p(y)=\infty\}=0$, it is sufficient to prove that

$$
q(x)=\int_{\{y, p(y)=\infty\}} g(x, y) \mu(d y) \equiv 0 .
$$

But since $q \leqq p$, the fact that (2) holds for $p$ implies that (2) holds for $q$. Hence (3) holds also for $q$. Let $B$ be the continuous homogeneous additive functional of $Y$ corresponding to $q$ by (3). Since by Proposition 3.1, $\left\{q\left(Y_{t \wedge \tau_{n}}\right), 0 \leqq t \leqq \infty, P_{x}\right\}$ is a martingale for all $n$ and $x \in \Omega_{q}$, we get $q(x)=E_{x} B_{\infty}=0$ for $x \in \Omega_{q}$, hence $q \equiv 0$. We finish the proof of this proposition by showing that $\left(2^{\prime}\right)$ implies (2). Let $x_{0} \in \Omega_{p}$, and assume that $\lim _{n \rightarrow \infty} n P_{x_{0}}\left\{\tau_{n}<\infty\right\}=0$. Assume first that $p$ is continuous (possibly infinite). If also $x_{1} \in \Omega_{p}$, then for some $n_{1}, x_{0}, x_{1} \in\left\{p<n_{1}\right\}$. For $n \geqq n_{1}$, the functions $n P .\left\{\tau_{n}<\infty\right\}$ are harmonic on the open set $\left\{p<n_{1}\right\}$ and we have by Harnack's inequality $n P_{x_{1}}\left\{\tau_{n}<\infty\right\} \leqq C \cdot n P_{x_{0}}\left\{\tau_{n}<\infty\right\}$ with $C<\infty$, which implies $\lim _{n \rightarrow \infty} n P_{x_{1}}\left\{\tau_{n}<\infty\right\}=0$. In the general case let $p=\sum p_{k}$ where the $p_{k}$ are continuous. Then $x_{0} \in \Omega_{p_{k}}$ and if $\tau_{n}^{(k)}=T_{\left\{p_{k}>n\right\}}$, then $\lim _{n \rightarrow \infty} n P_{x_{0}}\left\{\tau_{n}^{(k)}<\infty\right\}=0$. Therefore (2) and hence (3) hold for $p_{k}$. Let $A^{(k)}$ be the $A$ corresponding to $p_{k}$ by (3); then the processes $\left\{p_{k}\left(Y_{t}\right)+A_{t}^{(k)}, 0 \leqq t \leqq \infty, P_{x}\right\}$ are martingales for $x \in \Omega_{p_{k}}$. Since $\Omega_{p} \subseteq \bigcap_{k} \Omega_{p_{k}}$ we conclude by VII, T32(1) and VI, T20 in [5] that (2) holds for $p$.

REMARK. If the conditions of Proposition 3.2 hold for $p$, then (c) and the "energy formula," VII, T23 in [5] imply

$$
E_{x} A_{\infty}^{2}=2 E_{x} \int_{0}^{\infty} p\left(Y_{s}\right) d A_{s}=2 E_{x} \int_{0}^{\zeta} p\left(Y_{s}\right) d A_{s}=2 \int_{\Omega} p(y) g(x, y) \mu(d y) .
$$


On the other hand if $p_{1}(x)=2 \int p(y) g(x, y) \mu(d y) \not \equiv \infty$, then conditions (1)-(3) of Proposition 3.2 hold, since $p_{1}\left(x_{0}\right)<\infty$ implies $\lim _{n \rightarrow \infty} n^{2} P_{x_{0}}\left\{\tau_{n}<\infty\right\}=0$. This can be seen as follows. If $p_{1}\left(x_{0}\right)<\infty$, then clearly $x_{0} \in \Omega_{p}$. By the Frostman maximum principle, which says that the supremum of a potential equals its supremum on the support of the corresponding measure, we have for $x \in \Omega$,

$$
p(x) \leqq \int_{n / 2<p} g(x, y) \mu(d y)+\frac{n}{2} .
$$

Hence we get for $x \in\{p>n\}, 1 \leqq(2 / n) \int_{n / 2<p} g(x, y) \mu(d y)$, which implies for $x \in \Omega$, $P_{x}\left\{\tau_{n}<\infty\right\} \leqq(2 / n) \int_{n / 2<p} g(x, y) \mu(d y)$, because $P_{x}\left\{\tau_{n}<\infty\right\}$ is the equilibrium potential at $x$ of the open set $\{p>n\}$. We conclude

$$
n^{2} P_{x}\left\{\tau_{n}<\infty\right\} \leqq 4 \int_{n / 2<p} p(y) g(x, y) \mu(d y) .
$$

But the right side converges to 0 for $x=x_{0}$ because $\int_{\Omega} p(y) g\left(x_{0}, y\right) \mu(d y)<\infty$, which implies also $\mu\{y ; p(y)=\infty\}=0$.

As a consequence of Propositions 3.1 and 3.2 we obtain the following

Proposition 3.3. If $p(x)=\int g(x, y) \mu(d y)$ is a potential on $\Omega$, then there is $a$ uniquely determined continuous homogeneous additive functional $A$ of $Y$ (considered as process with state space $\Omega_{p}$ ) such that, for all $n$ and all $x \in \Omega_{p}$, the processes

$$
\left\{p\left(Y_{t \wedge \tau_{n}}\right)+A_{t \wedge \tau_{n}}, 0 \leqq t \leqq \infty, P_{x}\right\}
$$

are martingales. Moreover $E_{x} A_{\infty} \leqq p(x)$.

Proof. By VI, P3.6 in [1], there is a unique decomposition $\mu=\mu_{1}+\mu_{2}$ where $\mu_{1}$ does not charge any polar set and $\mu_{2}$ lives on a polar set. The proposition follows from the two preceding propositions.

REMARK. This proposition is the analogue of a theorem of $\mathrm{K}$. Ito and S. Watanabe about the representation of a nonnegative supermartingale as the sum of a local martingale and an increasing process.

We shall prove now two lemmas which will be useful.

LEMMA 3.4. If $p(x)=\int g(x, y) \mu(d y)$ is a potential on $\Omega$, then

$$
2 \int_{\Omega} p(y) g(x, y) \mu(d y)=p^{2}(x)+\int_{\Omega}|\operatorname{grad} p|^{2}(y) g(x, y) d y .
$$

Proof. Assume first that $p$ is bounded. Then $p^{2}$ is a distribution and we obtain (in distribution sense)

$$
\Delta\left(p^{2}\right)=2|\operatorname{grad} p|^{2}-4 p \mu .
$$

(It is not difficult to see that the application of the product rule in the preceding differentiation is legitimate. In particular, $|\operatorname{grad} p|^{2}$ is locally integrable, because for a bounded potential $p_{0}$ whose measure $\mu_{0}$ has compact support, the energy 
$\int_{\Omega}\left|\operatorname{grad} p_{0}\right|^{2}(x) d x=$ const $\int_{\Omega} p_{0}(x) \mu_{0}(d x)$ is finite.) If we now let $v=p_{1}-p^{2}$ with $p_{1}(x)=2 \int p(y) g(x, y) \mu(d y)$, then $\Delta v=-2|\operatorname{grad} p|^{2}$ (in distribution sense), which implies that $v=u$ a.e. (Lebesgue measure), where $u$ is superharmonic. Since $v$ is bounded, so is $u$, and hence the potential part of $u$, namely

$$
p_{2}(x)=\int|\operatorname{grad} p|^{2}(y) g(x, y) d y .
$$

Now let $w=p^{2}+p_{2}$. Then $\Delta w=-4 p \mu$. Since $w$ is lower semicontinuous and bounded and $w^{*} \equiv 0$, we have $w=p_{1}$.

In the general case let

$$
p_{n}(x)=p(x) \wedge n=\int g(x, y) \mu_{n}(d y) \quad(n \geqq 3) .
$$

Then $p_{n} \uparrow p$, and it is sufficient to prove:

$$
\begin{aligned}
\lim _{n \rightarrow \infty} \int\left|\operatorname{grad} p_{n}\right|^{2} g(x, y) d y & =\int|\operatorname{grad} p|^{2}(y) g(x, y) d y, \\
\lim _{n \rightarrow \infty} \int p_{n}(y) g(x, y) \mu_{n}(d y) & =\int p(y) g(x, y) \mu(d y) .
\end{aligned}
$$

(1) is proved as follows: $\operatorname{grad} p$ and $\operatorname{grad} p_{n}$ exist for all $x \notin N$ where $N \subseteq \Omega$ is a set of Lebesgue measure 0 ; moreover if $x \notin N$, then $\left|\operatorname{grad} p_{n}\right|^{2}(x) \leqq\left|\operatorname{grad} p_{n+1}\right|^{2}(x)$ $\leqq|\operatorname{grad} p|^{2}(x)$, equality occurring for sufficiently large $n$. (1) follows from the monotone covergence theorem.

(2) follows for any sequence $p_{n}(x)=\int g(x, y) \mu_{n}(d y) \uparrow p(x)$ from the remark after Proposition 3.2 and VII, T62 and T63 in [5].

ReMarK. The preceding lemma and the proof of (2) imply that for any sequence $p_{n}(x)=\int g(x, y) \mu_{n}(d y) \uparrow p(x),(1)$ is also true, for all $x \in \Omega_{p}$. (1) and (2) can be interpreted as analogues of a classical theorem on the convergence of the energy of potentials.

Lemma 3.5. Let $p(x)=\int g(x, y) \mu(d y)$ be a potential on $\Omega$ and assume that

$$
p_{1}(x)=2 \int p(y) g(x, y) \mu(d y) \not \equiv \infty
$$

or equivalently that $p_{2}(x)=\int|\operatorname{grad} p|^{2}(y) g(x, y) d y \not \equiv \infty$. Let $A$ be the continuous homogeneous additive functional associated with $p$ by Proposition 3.2. If we let $M_{t}=p\left(Y_{t}\right)+A_{t}$, then for all $x \in \Omega_{p_{1}}=\Omega_{p} \cap \Omega_{p_{2}}$, the processes

$$
\left\{M_{t}^{2}-\int_{0}^{t \wedge \xi}|\operatorname{grad} p|^{2}\left(Y_{s}\right) d s, 0 \leqq t \leqq \infty, P_{x}\right\}
$$

are martingales. 
Proof. By the remark following Proposition 3.2, $A$ is well defined by Proposition 3.2. Moreover, $E_{x} M_{\infty}^{2}=E_{x} A_{\infty}^{2}=p_{1}(x)<\infty$ if $x \in \Omega_{p_{1}}$. The functional

$$
\int_{0}^{t \wedge \xi}|\operatorname{grad} p|^{2}\left(Y_{s}\right) d s
$$

is also well defined, because $\operatorname{grad} p$ exists a.e. on $\Omega$ (w.r.t. Lebesgue measure); moreover,

$$
E_{x} \int_{0}^{t \wedge \zeta}|\operatorname{grad} p|^{2}\left(Y_{s}\right) d s \leqq p_{2}(x)<\infty \quad \text { if } x \in \Omega_{p_{2}} .
$$

We have $M_{t}^{2}=p_{1}\left(Y_{t}\right)-p_{2}\left(Y_{t}\right)+2 A_{t} p\left(Y_{t}\right)+A_{t}^{2}$. Now, for $x \in \Omega_{p_{1}}$,

(1) $\left\{p_{1}\left(Y_{t}\right)+2 \int_{0}^{t} p\left(Y_{s}\right) d A_{s}, 0 \leqq t \leqq \infty, P_{x}\right\}$ is a martingale because $2 \int_{0}^{t} p\left(Y_{s}\right) d A_{s}$ is a continuous homogeneous additive functional of $Y$ and $2 E_{x} \int_{0}^{\infty} p\left(Y_{s}\right) d A_{s}$ $=E_{x} A_{\infty}^{2}=p_{1}(x)$.

(2) $\left\{p_{2}\left(Y_{t}\right)+\int_{0}^{t \wedge \zeta}|\operatorname{grad} p|^{2}\left(Y_{s}\right) d s, 0 \leqq t \leqq \infty, P_{x}\right\}$ is a martingale.

(3) $\left\{A_{t} p\left(Y_{t}\right)+\frac{1}{2} A_{t}^{2}-\int_{0}^{t} p\left(Y_{s}\right) d A_{s}, 0 \leqq t \leqq \infty, P_{x}\right\}$ is a martingale, because

$$
\begin{aligned}
E_{x}\left\{\frac{1}{2} A_{\infty}^{2}-\int_{0}^{\infty} p\left(Y_{s}\right) d A_{s} \mid \mathfrak{F}_{t}\right\} & \\
= & \frac{1}{2} A_{t}^{2}+A_{t} E_{Y_{t}} A_{\infty}+\frac{1}{2} E_{Y_{t}} A_{\infty}^{2}-\int_{0}^{t} p\left(Y_{s}\right) d A_{s}-E_{Y_{t}} \int_{0}^{\infty} p\left(Y_{s}\right) d A_{s} \\
& =\frac{1}{2} A_{t}^{2}+A_{t} p\left(Y_{t}\right)-\int_{0}^{t} p\left(Y_{s}\right) d A_{s} .
\end{aligned}
$$

The lemma follows from (1), (2), (3).

THEOREM 3.6. Let $p(x)=\int g(x, y) \mu(d y)$ be a potential on $\Omega$, let

and let

$$
S^{2}(\omega)=\int_{0}^{\zeta}|\operatorname{grad} p|^{2}\left(Y_{s}\right) d s
$$

$$
E_{x} S^{2}=\int|\operatorname{grad} p|^{2}(y) g(x, y) d y=p_{2}(x) .
$$

(a) If $x \in \Omega_{p}$, then $S^{2}<\infty, P_{x}$-a.e.

(b) If $x \in \Omega_{p}$, then $S^{2}\left(p, \pi_{n}\right) \rightarrow S^{2}$ in $P_{x}$-probability for every sequence $\left\{\pi_{n}\right\}$ such that $\left\|\pi_{n}\right\| \rightarrow 0$ and $t_{k_{n}}^{(n)} \rightarrow \infty$.

(c) If $x \in \Omega_{p} \cap \Omega_{p_{2}}$, then $S^{2}\left(p, \pi_{n}\right) \rightarrow S^{2}$ in $L_{1}\left(P_{x}\right)$-norm for every sequence $\left\{\pi_{n}\right\}$ such that $\left\|\pi_{n}\right\| \rightarrow 0$ and $t_{k_{n}}^{(n)} \rightarrow \infty$.

(d) If $x \in \Omega_{p} \cap \Omega_{p_{2}}$ and if $\int|\operatorname{grad} p|^{4} g(x, y) d y<\infty$, then $S^{2}\left(p, \pi_{n}\right) \rightarrow S^{2}, P_{x}$-a.e. for every sequence $\left\{\pi_{n}\right\}$ such that $\sum\left\|\pi_{n}\right\|<\infty, t_{k_{n}}^{(n)} \rightarrow \infty$.

Proof. Let $A$ be the continuous homogeneous additive functional associated with $p$ by Proposition 3.3. Let $M_{t}=p\left(Y_{t}\right)+A_{t}$. Then

$$
\begin{aligned}
S^{2}\left(p, \pi_{n}\right)= & \sum_{j=0}^{k_{n}-1}\left(M_{t_{j+1}^{(n)}}-M_{t_{j}^{(n)}}\right)^{2}+\sum_{j=0}^{k_{n}-1}\left(A_{t_{+1}^{(n)}}-A_{t_{j}^{(n)}}\right)^{2} \\
& -2 \sum_{j=0}^{k_{n}-1}\left(M_{t_{j+1}^{(n)}}-M_{t_{j}^{(n)}}\right)\left(A_{t_{j+1}^{(n)}}-A_{t_{j}^{(n)}}\right) .
\end{aligned}
$$


If $x \in \Omega_{p}$, and $\left\|\pi_{n}\right\| \rightarrow 0$, then

and

$$
\sum_{j=0}^{k_{n}-1}\left(A_{t_{j+1}^{(n)}}-A_{t_{j}^{(n)}}\right)^{2} \rightarrow 0 \quad P_{x^{-} \text {-a.e. }}
$$

$$
\sum_{j=0}^{k_{n}-1}\left(M_{t_{j+1}^{(n)}}-M_{t_{j}^{(n)}}\right)\left(A-A_{t_{j+1}^{(n)}}\right) \rightarrow 0 \quad P_{x^{-}} \text {-a.e. }
$$

This follows from the fact that the first sum is majorized by $A_{\infty} \cdot \sup _{j}\left(A_{t_{j+1}^{(n)}}-A_{t_{j}^{(n)}}\right)$ and the absolute value of the second one is majorized by $A_{\infty} \cdot \sup _{j}\left|M_{t_{j+1}^{(n)}}-M_{t_{j}^{(n)}}\right|$ and $P_{x}$-a.e., both $A_{\text {. }}(\omega)$ and $M_{\text {. }}(\omega)$ are continuous and hence uniformly continuous on $[0, \infty]$.

If $x \in \Omega_{p} \cap \Omega_{p_{2}}$ and $\left\|\pi_{n}\right\| \rightarrow 0$, then also

and

$$
E_{x}\left\{\sum_{j=0}^{k_{n}-1}\left(A_{t_{j+1}^{(n)}}-A_{t_{j}^{(n)}}\right)^{2}\right\} \rightarrow 0
$$

$$
E_{x} \mid \sum_{j=0}^{k_{n}-1}\left(M_{t_{j+1}^{(n)}}-M_{t_{j}^{(n)}}\right)\left(A_{t_{j+1}^{(n)}}-A_{\left.t_{j}^{(n)}\right)} \mid \rightarrow 0 .\right.
$$

This follows from the fact that the integrands converge to $0 P_{x}$-a.e. and are majorized by $A_{\infty}^{2}$ and $A_{\infty}\left(A_{\infty}+\sup _{t} p\left(Y_{t}\right)\right)$ respectively; from p. 142 in [5] we have $E_{x}\left\{\sup _{t} p\left(Y_{t}\right)\right\}^{2} \leqq 4 E_{x} A_{\infty}^{2}=4 p_{1}(x)$.

We therefore have to discuss

$$
S^{2}\left(M, \pi_{n}\right)=\sum_{j=0}^{k_{n}-1}\left(M_{t_{j+1}^{(n)}}-M_{t_{j}^{(n)}}\right)^{2}
$$

Proof of (a) and (b). Let $M_{t}^{(n)}=M_{t \wedge \tau_{n}}$ for $0 \leqq t \leqq \infty$ where $\tau_{n}=T_{\{p>n\}}$. Then by Proposition 3.3, $\left\{M_{t}^{(n)}, 0 \leqq t \leqq \infty, P_{x}\right\}$ is a martingale for $x \in \Omega_{p}$. We shall prove that $S^{2}\left(M^{(n)}, \pi_{k}\right) \rightarrow \int_{0}^{\tau_{n} \wedge \zeta}|\operatorname{grad} p|^{2}\left(Y_{s}\right) d s$ in $P_{x}$-probability. This is trivial if $x \in\{p>n\}$; so we may assume that $x \in\{p \leqq n\}$. In view of Lemma 2.1 it is sufficient to prove that

$$
\left\{M_{t}^{(n)^{2}}-\int_{0}^{t \wedge \tau_{n} \wedge \zeta}|\operatorname{grad} p|^{2}\left(Y_{s}\right) d s, 0 \leqq t \leqq \infty, P_{x}\right\}
$$

is a martingale. This is seen as follows: Let $p_{n}=p \wedge n$, and let $A^{(n)}$ be the continuous homogeneous additive functional associated with $p_{n}$, and let $N_{t}^{(n)}=p_{n}\left(Y_{t}\right)+A_{t}^{(n)}$. Then by Lemma 3.5,

$$
\left\{N_{t}^{(n)^{2}}-\int_{0}^{t \wedge \zeta}\left|\operatorname{grad} p_{n}\right|^{2}\left(Y_{s}\right) d s, 0 \leqq t \leqq \infty, P_{x}\right\}
$$

is a martingale, hence

$$
\left\{N_{t \wedge \tau_{n}}^{(n)^{2}}-\int_{0}^{t \wedge \tau_{n} \wedge \zeta}\left|\operatorname{grad} p_{n}\right|^{2}\left(Y_{s}\right) d s, 0 \leqq t \leqq \infty, P_{x}\right\}
$$


is a martingale. But $N_{t \wedge \tau_{n}}^{(n)}=M_{t}^{(n)}, P_{x}$-a.e., because $P_{x}$-a.e., $p_{n}\left(Y_{t \wedge \tau_{n}}\right)=N_{t \wedge \tau_{n}}^{(n)}-A_{t \wedge \tau_{n}}^{(n)}$, $p_{n}\left(Y_{t \wedge \tau_{n}}\right)=p\left(Y_{t \wedge \tau_{n}}\right)=M_{t}^{(n)}-A_{t \wedge \tau_{n}}$ and the decomposition of $p_{n}\left(Y_{t \wedge \tau_{n}}\right)$ is unique. Also

$$
\int_{0}^{t \wedge \tau_{n} \wedge \zeta}\left|\operatorname{grad} p_{n}\right|^{2}\left(Y_{s}\right) d s=\int_{0}^{t \wedge \tau_{n} \wedge \zeta}|\operatorname{grad} p|^{2}\left(Y_{s}\right) d s .
$$

Hence $\left\{M_{t}^{(n)^{2}}-\int_{0}^{t \wedge \tau_{n} \wedge \xi}|\operatorname{grad} p|^{2}\left(Y_{s}\right) d s, 0 \leqq t \leqq \infty, P_{x}\right\}$ is a martingale, and

$$
S^{2}\left(M^{(n)}, \pi_{k}\right) \rightarrow \int_{0}^{\tau_{n} \wedge \zeta}|\operatorname{grad} p|^{2}\left(Y_{s}\right) d s
$$

in $P_{x}$-probability as $k \rightarrow \infty$, if $x \in \Omega_{p}$.

For the remainder of the proof we use an argument of the proof of Theorem 6.2 in [6]. If $x \in \Omega_{p}$, then

$$
P_{x}\left\{\left|S^{2}\left(M, \pi_{k}\right)-S^{2}\left(M^{(n)}, \pi_{k}\right)\right|>\varepsilon\right\} \leqq P_{x}\left\{\tau_{n}<\infty\right\} \rightarrow 0
$$

uniformly in $k$. Because of

$$
\begin{aligned}
P_{x}\left\{\left|S^{2}\left(M, \pi_{k_{2}}\right)-S^{2}\left(M, \pi_{k_{1}}\right)\right|>\varepsilon\right\} \leqq & P_{x}\left\{\left|S^{2}\left(M, \pi_{k_{2}}\right)-S^{2}\left(M^{(n)}, \pi_{k_{2}}\right)\right|>\varepsilon / 3\right\} \\
& +P_{x}\left\{\left|S^{2}\left(M^{(n)}, \pi_{k_{2}}\right)-S^{2}\left(M^{(n)}, \pi_{k_{1}}\right)\right|>\varepsilon / 3\right\} \\
& +P_{x}\left\{\left|S^{2}\left(M^{(n)}, \pi_{k_{1}}\right)-S^{2}\left(M, \pi_{k_{1}}\right)\right|>\varepsilon / 3\right\}
\end{aligned}
$$

we conclude that there is a real-valued random variable $\xi$ such that $S^{2}\left(M, \pi_{k}\right) \rightarrow \xi$ in $P_{x}$-probability as $k \rightarrow \infty$. From (X) and (XX) we obtain

$$
P_{x}\left\{\left.\left|\xi-\int_{0}^{\tau_{n} \wedge \xi}\right| \operatorname{grad} p\right|^{2}\left(Y_{s}\right) d s \mid>\varepsilon\right\} \leqq P_{x}\left\{\tau_{n}<\infty\right\} \rightarrow 0
$$

and therefore $\xi=\int_{0}^{\zeta}|\operatorname{grad} p|^{2}\left(Y_{s}\right) d s=S^{2}$. This proves (a) and (b).

Proof of (c) and (d). If $\Omega_{p_{1}}=\Omega_{p} \cap \Omega_{p_{2}} \neq \varnothing$, then $\mu$ does not charge any polar set and $\left\{M_{t}, 0 \leqq t \leqq \infty, P_{x}\right\}$ is a martingale for $x \in \Omega_{p}$. Now (c) follows from Lemmas 2.1 and 3.5, (d) from Lemmas 2.3 and 3.5.

REMARK. The random variable $S^{2}$ is in general not a constant on $Y$-paths converging to a fixed boundary point. For example let $\Omega=R_{n}(n>2)$ and let $p$ be the potential of a measure living on a polar set $\subseteq R_{n}$. Here $\partial \Omega=\{\infty\}, E_{x} S^{2}=p_{2}(x)$ $\equiv \infty$, whereas, for $x \in \Omega_{p}, S^{2}<\infty P_{x}$-a.e.

4. Quadratic variation of harmonic functions. We start by introducing some notations. Let $\Omega_{n} \uparrow \Omega, \bar{\Omega}_{n}$ compact $\subseteq \Omega$; let $\zeta_{n}$ be the life-time of $Y$ in $\Omega_{n}$.

If $h$ is harmonic on $\Omega$ and $r \geqq 1$, let $\|h\|_{r}^{r}(x)=\sup _{\Omega_{n} \ni x} E_{x}\left|h\left(Y_{\zeta_{n}}\right)\right|^{r}$. The following facts are well known:

(1) The value of $\|h\|_{r}(x)$ is independent of the particular sequence $\left\{\Omega_{n}\right\}$; moreover $\left\|h_{r}\right\|(x)<\infty$ for all $x$ or $\|h\|_{r}(x) \equiv \infty$.

(2) $\|h\|_{1}<\infty$ iff $h$ is the difference of two positive harmonic functions. In this case $h$ has a fine boundary function $h^{*}$. 
(3) If $r>1$, then $\|h\|_{r}<\infty$ iff $h$ is the Dirichlet solution corresponding to an $\mathscr{L}_{r}$-boundary function $h^{*}\left(\mathscr{L}_{r}\right.$ w.r.t. harmonic measure $\omega$ on $\left.\partial \Omega\right)$. In this case:

$$
\|h\|_{r}^{r}(x)=E_{x}\left|h^{*}\left(Y_{\zeta}\right)\right|^{r}=\int_{\partial \Omega}\left|h^{*}(y)\right|^{r} \omega(x, d y) .
$$

REMARK. If $h$ is harmonic on $\Omega$, then $\|h\|_{2}<\infty$ iff $\int_{\Omega}|\operatorname{grad} h|^{2}(y) g(x, y) d y<\infty$ for some $x \in \Omega$ (and hence for all $x \in \Omega$ ). This follows from

$$
\|h\|_{2}^{2}(x)-h^{2}(x)=\int_{\Omega}|\operatorname{grad} h|^{2}(y) g(x, y) d y .
$$

The preceding equation follows from the Riesz decomposition of the restriction of the subharmonic function $h^{2}$ to $\Omega_{n}$, where $\Omega_{n} \uparrow \Omega, \bar{\Omega}_{n}$ compact $\subseteq \Omega$.

THEOREM 4.1. Let $h$ be harmonic on $\Omega$ and let $S^{2}=\int_{0}^{\zeta}|\operatorname{grad} h|^{2}\left(Y_{s}\right) d s$.

(a) If $h$ is the difference of two positive harmonic functions or equivalently if $\|h\|_{1}<\infty$, then for all $x \in \Omega, S^{2}<\infty P_{x^{-}}$a.e. and $S^{2}\left(h, \pi_{n}\right) \rightarrow S^{2}$ in $P_{x}$-probability for $\left\|\pi_{n}\right\| \rightarrow 0$ and $t_{k_{n}}^{(n)} \rightarrow \infty$.

(b) For $r>1$, there are positive finite numbers $\alpha_{r}$ and $\beta_{r}$, independent of $\Omega, h$ and $x$, such that

$$
\alpha_{r} E_{x} S^{r} \leqq\|h-h(x)\|_{r}^{r}(x) \leqq \beta_{r} E_{x} S^{r}
$$

If $\|h\|_{r}<\infty$ or equivalently $E$. $S^{r}<\infty$ then for all $x \in \Omega, S\left(h, \pi_{n}\right) \rightarrow S$ in $L_{r}\left(P_{x}\right)$-norm for $\left\|\pi_{n}\right\| \rightarrow 0$ and $t_{k_{n}}^{(n)} \rightarrow \infty$.

(c) If $E_{x} S^{2}=\int|\operatorname{grad} h|^{2}(y) g(x, y) d y<\infty$ (or equivalently if $\|h\|_{2}<\infty$ ) and if $\int|\operatorname{grad} h|^{4}(y) g(x, y) d y<\infty$, then for all $x \in \Omega, S^{2}\left(h, \pi_{n}\right) \rightarrow S^{2}, P_{x^{-}}$a.e. for $\sum\left\|\pi_{n}\right\|<\infty$ and $t_{k_{n}}^{(n)} \rightarrow \infty$.

REMARK. The integrals in (c) converge for all $x \in \Omega$ or for none.

Proof. (a) Let $\tau_{n}=T_{\{|n|>n\}}$. Because $P_{x}$-a.e., $h\left(Y_{t}\right)$ is finite and continuous on $[0, \infty], P_{x}\left\{\tau_{n}<\infty\right\} \rightarrow 0$ for all $x \in \Omega$. Let $M_{t}^{(n)}=h\left(Y_{t \wedge \tau_{n}}\right)$. We show first that, for all $x \in \Omega, S^{2}\left(M^{(n)}, \pi_{k}\right) \rightarrow \int_{0}^{\zeta \wedge \tau_{n}}|\operatorname{grad} h|^{2}\left(Y_{s}\right) d s$ in $P_{x}$-probability. This is trivial if $|h(x)| \geqq n$. We may assume therefore that $|h(x)|<n$. It is clear that

$$
\left\{M_{t}^{(n)}, 0 \leqq t \leqq \infty, P_{x}\right\}
$$

is a continuous bounded martingale. In view of Lemma 2.1 it is sufficient to show that for all $x \in \Omega$,

$$
\left\{M_{t}^{(n)^{2}}-\int_{0}^{t \wedge \tau_{n} \wedge \zeta}|\operatorname{grad} h|^{2}\left(Y_{s}\right) d s, 0 \leqq t \leqq \infty, P_{x}\right\}
$$

is a martingale. Now if $\Omega_{n}$ is the component of the open set $\{|h|<n\}$ which contains $x$ and if $g_{n}$ is its Green function, then, for $y \in \Omega_{n}$,

$$
\begin{aligned}
E_{y} \int_{0}^{\tau_{n} \wedge \zeta}|\operatorname{grad} h|^{2}\left(Y_{s}\right) d s & =\int_{\Omega_{n}}|\operatorname{grad} h|^{2}(z) g_{n}(y, z) d z \\
& =H_{h^{2}}^{\Omega_{n}}(y)-h^{2}(y)<\infty,
\end{aligned}
$$


where $H_{h^{2}}^{\Omega_{n}}$ is the harmonic function on $\Omega_{n}$ which has as boundary function the restriction of $h^{2}$. This implies that (X) is a martingale. To finish the proof of (a) we observe that for $x \in \Omega$,

$$
P_{x}\left\{\left|S^{2}\left(M^{(n)}, \pi_{k}\right)-S^{2}\left(M, \pi_{k}\right)\right|>\varepsilon\right\} \leqq P_{x}\left\{\tau_{n}<\infty\right\} \rightarrow 0,
$$

uniformly in $k$, and repeat the last argument in the proof of (a) and (b) of Theorem 3.6.

(b) If $\|h\|_{r}<\infty$, then $\left\{h\left(Y_{t}\right), 0 \leqq t \leqq \infty\right\}$ is a continuous martingale and

$$
E_{x}\left|h\left(Y_{\infty}\right)\right|^{r}=E_{x}\left|h^{*}\left(Y_{\zeta}\right)\right|^{r}<\infty .
$$

The $L_{r}$-convergence of $S^{2}\left(h, \pi_{n}\right)$ and the inequality for the moments follow from Theorem 6.2 in [6].

If $\|h\|_{r}=\infty$, the left inequality for the moments is trivial; but it is also not difficult to see that $\|h\|_{r}=\infty$ implies $E_{x} S^{r}=\infty$. Let $\Omega_{n} \uparrow \Omega, \bar{\Omega}_{n}$ compact $\subseteq \Omega$, $x \in \Omega_{n}$ and let $\zeta_{n}$ be the life-time of $Y$ in $\Omega_{n}$. Then $h$ is bounded on $\Omega_{n}$ and

$$
E_{x}\left|h\left(Y_{\zeta_{n}}\right)\right|^{r} \leqq \beta_{r} E_{x}\left(\int_{0}^{\zeta_{n}}|\operatorname{grad} h|^{2}\left(Y_{s}\right) d s\right)^{r / 2} .
$$

Letting $n \rightarrow \infty$, we get $\infty=E_{x} S^{r}$.

(c) Here the processes $\left\{h\left(Y_{t}\right), 0 \leqq t \leqq \infty, P_{x}\right\}$ and

$$
\left\{h^{2}\left(Y_{t}\right)-\int_{0}^{t \wedge \zeta}|\operatorname{grad} h|^{2}\left(Y_{s}\right) d s, 0 \leqq t \leqq \infty, P_{x}\right\}
$$

are martingales for $x \in \Omega$, and the assertion follows from Lemma 2.3.

REMARK. If we let $r=2 n$ in (b) of the preceding theorem then we get estimates for harmonic functions which can be formulated in strictly nonprobabilistic language. Recall that the constants $\alpha_{2 n}$ and $\beta_{2 n}$ are independent of $\Omega$.

5. Generalization of a formula by $\mathbf{K}$. Ito. We are now able to give a generalization of a classical formula by $\mathrm{K}$. Ito. According to Ito we have for a function $u \in C^{2}\left(R_{n}\right)$, for all $x \in R_{n}, P_{x}$-a.e.

$$
u\left(Y_{t}\right)-u\left(Y_{0}\right)=P_{x}-\int_{0}^{t} \operatorname{grad} u\left(Y_{s}\right) \cdot d Y_{s}+\frac{1}{2} \int_{0}^{t}(\Delta u)\left(Y_{s}\right) d s
$$

Here $Y$ is Brownian motion on $R_{n}$. In the following we denote by $Y$ again Brownian motion on a Green domain $\Omega \subseteq R_{n}$, stopped upon reaching $\partial \Omega$.

TheOREM 5.1. (a) If $p$ is a potential on $\Omega$ and $A$ the continuous homogeneous additive functional corresponding to $p$ by Proposition 3.3 then, for $x \in \Omega_{p}, P_{x}$-a.e.,

$$
p\left(Y_{t}\right)-p\left(Y_{0}\right)=P_{x}-\int_{0}^{t \wedge \zeta} \operatorname{grad} p\left(Y_{s}\right) d Y_{s}-A_{t}
$$

(b) If $h$ is a harmonic function on $\Omega$ such that $\|h\|_{1}<\infty$, then for $x \in \Omega, P_{x}$-a.e.,

$$
h\left(Y_{t}\right)-h\left(Y_{0}\right)=P_{x}-\int_{0}^{t \wedge \zeta} \operatorname{grad} h\left(Y_{s}\right) \cdot d Y_{s}
$$


An immediate consequence of this theorem is the following

COROLlaRY 5.2. Let $u$ be the difference of two positive superharmonic functions on $\Omega$. If $|u(x)|<\infty$, then $P_{x}$-a.e.

$$
u\left(Y_{t}\right)-u\left(Y_{0}\right)=P_{x}-\int_{0}^{t \wedge \zeta} \operatorname{grad} u\left(Y_{s}\right) \cdot d Y_{s}-C_{t}
$$

where $C$ is the difference of two (nonnegative) continuous homogeneous additive functionals of $Y$ (with state space $\{x \in \Omega \cup \partial \Omega ;|u(x)|<\infty\}$ ).

Proof of Theorem 5.1(a).

(1) We assume first that $p \in C^{2}(\Omega)$. Let $\Omega_{k} \uparrow \Omega, \Omega_{k}$ compact $\subseteq \Omega, x \in \Omega_{k}$. Denote by $\zeta_{k}$ the life-time of $Y$ in $\Omega_{k}$. It is easy to see that the classical Ito formula

$$
p\left(Y_{t \wedge \zeta_{k}}\right)-p(x)=P_{x}-\int_{0}^{t \wedge \zeta_{k}} \operatorname{grad} p\left(Y_{s}\right) \cdot d Y_{s}+\frac{1}{2} \int_{0}^{t \wedge \zeta_{k}} \Delta p\left(Y_{s}\right) d s \quad P_{x} \text {-a.e. }
$$

is valid. But by Theorem 3.6(a), $\int_{0}^{\zeta}|\operatorname{grad} p|^{2}\left(Y_{s}\right) d s<\infty P_{x}$-a.e. Hence we obtain (x) with $A_{t}=-\frac{1}{2} \int_{0}^{t \wedge \zeta} \Delta p\left(Y_{s}\right) d s$ by taking in (o) the limits in $P_{x}$-probability as $k \rightarrow \infty$.

(2) Assume now that $p$ is a bounded potential on $\Omega$. We shall see first that for any nondecreasing sequence of potentials $p_{n} \uparrow p$, we have $\operatorname{grad} p_{n_{k}} \rightarrow \operatorname{grad} p$ a.e. (w.r.t. Lebesgue measure) for a subsequence $p_{n_{k}}$ : Let $B$ be any closed ball in $\Omega$, and let $p_{n}^{\prime}, p^{\prime}$ be the potentials obtained from $p_{n}, p$ by a "sweeping out" process with respect to $B$. The potentials $p_{n}^{\prime}, p^{\prime}$ are bounded, and the associated measures (living on $B$ ) have finite total mass. Therefore $p_{n}^{\prime}, p^{\prime}$ have finite energy; and since $p_{n}^{\prime} \uparrow p^{\prime}$, we get $\int_{\Omega}\left|\operatorname{grad}\left(p_{n}^{\prime}-p^{\prime}\right)\right|^{2}(x) d x \rightarrow 0$. But on $B, p_{n}^{\prime}=p_{n}$ and $p^{\prime}=p$, and we get $\operatorname{grad} p_{n_{k}} \rightarrow \operatorname{grad} p$ a.e. in the interior of $B$, for some sequence $n_{k}$. The rest follows from a diagonal argument. Now let $p_{n_{s}}$ be a nondecreasing sequence of potentials such that $p_{n} \in C^{2}(\Omega), p_{n} \uparrow p$. We may assume that $\operatorname{grad} p_{n} \rightarrow \operatorname{grad} p$ a.e. on $\Omega$. Now firstly, (x) is valid for $p_{n}$. Secondly, $A_{t}^{(n)}=-\frac{1}{2} \int_{0}^{t \wedge \zeta} \Delta p_{n}\left(Y_{s}\right) d s \rightarrow A_{t}$ in $L_{2}\left(P_{x}\right)$-norm because, by VII, T36 in [5], $E_{x}\left\{A_{\infty}^{(n)}-A_{\infty}\right\}^{2} \rightarrow 0$ and by Proposition (3.3) $A_{t}^{(n)}=E_{x}\left\{A_{\infty}^{(n)} \mid \mathfrak{F}_{t}\right\}-p_{n}\left(Y_{t}\right)$ and $A_{t}=E_{x}\left\{A_{\infty} \mid \mathfrak{F}_{t}\right\}-p\left(Y_{t}\right), P_{x}$-a.e. We conclude therefore that $P_{x}-\int_{0}^{t \wedge \zeta} \operatorname{grad} p_{n}\left(Y_{s}\right) \cdot d Y_{s}$ converges in $L_{2}\left(P_{x}\right)$-norm. If we denote by $q$ the transition function of $Y$ on $\Omega$ and let $g_{t}(x, y)=\int_{0}^{t} q_{s}(x, y) d s$, this implies

$$
\begin{aligned}
\sup _{k} \int_{\Omega} \mid \operatorname{grad}( & \left.p_{n+k}-p_{n}\right)\left.\right|^{2}(y) g_{t}(x, y) d y \\
\quad & \sup _{k} E_{x}\left\{\int_{0}^{t \wedge \zeta} \operatorname{grad} p_{n+k}\left(Y_{s}\right) \cdot d Y_{s}-\int_{0}^{t \wedge \zeta} \operatorname{grad} p_{n}\left(Y_{s}\right) \cdot d Y_{s}\right\}^{2} \\
& \rightarrow 0 .
\end{aligned}
$$

But since $\operatorname{grad} p_{n} \rightarrow \operatorname{grad} p$ a.e. on $\Omega$, we obtain

$$
\int_{\Omega}\left|\operatorname{grad}\left(p-p_{n}\right)\right|^{2}(y) g_{t}(x, y) d y \rightarrow 0 .
$$


The left side equals

$$
E_{x}\left\{\int_{0}^{t \wedge \zeta} \operatorname{grad} p\left(Y_{s}\right) \cdot d Y_{s}-\int_{0}^{t \wedge \zeta} \operatorname{grad} p_{n}\left(Y_{s}\right) \cdot d Y_{s}\right\}^{2}
$$

We have therefore $\int_{0}^{t \wedge \zeta} \operatorname{grad} p_{n}\left(Y_{s}\right) \cdot d Y_{s} \rightarrow \int_{0}^{t \wedge \zeta} \operatorname{grad} p\left(Y_{s}\right) \cdot d Y_{s}$ in $L_{2}\left(P_{x}\right)$-norm. We conclude that $(\mathrm{x})$ is valid for $p$.

(3) If finally $p$ is an arbitrary potential on $\Omega$, let $p_{n}=p \wedge n$. Then, for all $x \in \Omega$,

$$
p_{n}\left(Y_{t}\right)-p_{n}(x)=\int_{0}^{t \wedge \xi} \operatorname{grad} p_{n}\left(Y_{s}\right) \cdot d Y_{s}-A_{t}^{(n)} \quad P_{x} \text {-a.e. }
$$

where $A^{(n)}$ is the continuous homogeneous additive functional corresponding to $p_{n}$

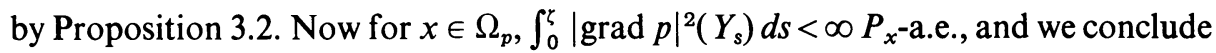
from $\left|\operatorname{grad} p_{n}\right|^{2} \uparrow|\operatorname{grad} p|^{2}$ a.e. (w.r.t. Lebesgue measure) that

$$
\int_{0}^{t \wedge \zeta} \operatorname{grad} p_{n}\left(Y_{s}\right) \cdot d Y_{s} \rightarrow \int_{0}^{t \wedge \zeta} \operatorname{grad} p\left(Y_{s}\right) \cdot d Y_{s}
$$

in $P_{x}$-probability. Moreover if the measure corresponding to $p$ does not charge any polar set and if $A$ is the continuous homogeneous additive functional corresponding to $p$ by Proposition 3.2, then $A_{t}^{(n)} \rightarrow A_{t}$ in $P_{x}$-probability for $x \in \Omega_{p}$. On the other hand, if the measure corresponding to $p$ lives on a polar set, then $A_{t}^{(n)} \rightarrow 0$ in $P_{x}$-probability for $x \in \Omega_{p}$. In either case we obtain (x) by taking in (+) the limit in $P_{x}$-probability as $n \rightarrow \infty$; for general $p$, we obtain (x) by using the decomposition in the proof of Proposition 3.3.

Proof of Theorem 5.1(b). The proof follows by the same argument as in the first part of the preceding proof.

\section{REFERENCES}

1. R. M. Blumenthal and R. K. Getoor, Markov processes and potential theory, Academic Press, New York, 1968.

2. K. È. Dambis, On the decomposition of continuous submartingales, Teor. Verojatnost. i Primenen. 10 (1965), 438-448. (Russian) MR 34 \#2052.

3. J. L. Doob, Stochastic processes, Wiley, New York, 1953. MR 15, 445.

4. D. L. Fisk, Sample quadratic variation of sample continuous, second order martingales, Z. Wahrscheinlichkeitstheorie und Verw. Gebiete 6 (1966), 273-278. MR 35 \#1078.

5. P. A. Meyer, Probability and potentials, Blaisdell, Waltham, Mass., 1966. MR 34 \#5119.

6. P. W. Millar, Martingale integrals, Trans. Amer. Math. Soc. 133 (1968), 145-166. MR 37 \#2308.

7. E. Wong and M. Zakai, The oscillation of stochastic integrals, Z. Wahrscheinlichkeitstheorie und Verw. Gebiete 4 (1965), 103-112. MR 32 \#3112.

8. M. Brelot, Éléments de la théorie classique du potentiel, Les cours de Sorbonne, Centre de Documentation Univ., Paris, 1959. MR 21 \#5099.

9. L. Naïm, Sur le rôle de la frontière de R. S. Martin dans la théorie du potentiel, Ann. Inst. Fourier Grenoble 7 (1957), 183-281. MR 20 \#6608.

UNIVERSity of British COLUMBia, VANCOUVER, CANADA 